IV.

Aus den pharmakologisehen Instituten zu Straßburg und Wien.

\title{
Ueber das Verhalten des Chinolins im Tierkörper.
}

\author{
(Erste Mitteilung.) \\ Von \\ Ur. Hermann Fiühner.
}

Während das Kilogramm Chininsulfat, nach Flückigers Angaben, im Jahre 1876 noch 195 Mk. kostete, war der Preis desselben vier Jahre später (1880) auf $430 \mathrm{Mk}$. gestiegen, und es erscheint somit verständlich, daß gerade damals eifrig nach einem billigeren Ersatz für dies wertvolle Alkaloid gesucht wurde.

Der Gedanke lag nahe, zu prüfen, ob nicht das Chinolin, welches schon im Jahre 1842 von K. F. Gerhardt ${ }^{1}$ ) aus einem Chinaalkaloide, dem Cinchonin, auf dem Wege der Kalischmelze erhalten worden war, der Träger der Arzneiwirkung des Chinins sei, da es aus diesem gleichfalls durch Behandeln mit Ätzalkalien gewonnen werden kann.

Von dieser Vorstellung ausgehend untersuchte J. Donath ${ }^{2}$ ) im Jahre 1881 die Wirkung des Chinolins auf den Tierkörper, und als ihm gelang, nachzuweisen, daß es in der Tat die Körpertemperatur herabsetzt, wurde allseitig mit großen Hoffnungen an die klinische Prüfung ${ }^{3}$ ) dieses Produktes gegangen, das seit dem Jahre 1880 durch die berühmte Skraupsche Synthese bequem zugänglich geworden war.

Doch sehr rasch schon erwies sich das Chinolin als therapeutisch völlig unbrauchbar, denn neben unsicherer Entfieberung verursachte es in größeren Dosen Kollaps und namentlich häufig Er-

1) Zur Chemie des Chinolins vergleiche: A. Reissert, Das Chinolin und und seine Derivate. Braunschweig 1889 und A. Calm u. K. v. Buchka, Die Chemie des Pyridins und seiner Derivate. Braunschweig 1889-1891.

2) J. Donath, Physiol. u. physiol.-chemische Wirkungen des Chinolins. Ber. d. Dentsch. chem. Ges. 14, 178 u. 1769 (1881).

3) Klinische Untersuchungen von Sakowsky (1880), v. Jaksch (1881), Löw y (1881), Brieger (1882), Nahmmacher (1882), Koch (1882), Kupke (1882), Bodenhausen (1882), Seifert (1883). 
brechen, das sich bis zu Magenblutungen steigern konnte. Gegen Malaria fehlt ihm jegliche spezifische Wirkung, ebenso wie seinen gleichfalls kaum mehr gebrauchten Derivaten, von welchen hier nur Kairolin (Tetrahydrochinolinmethylschwefelsäure), Kair in (Tetrahydro-0-oxychinolinmethylehlorhydrat), Thall in (Tetrahydro-p-chinanisol) und Analgen (o-Äthoxy-ana-benzoylamidochinolin) genannt seien.

Neben der temperaturherabsetzenden Wirkung des Chinolins hatte schon Donath seine stark antiseptischen und antizymotischen Eigenschaften hervorgehoben, und die fußend auf dieser Beobachtung hergestellten chemischen Präparate finden noch immer in der Wundbehandlung ansgedehnte Verwendung. Die wichtigsten hierhergehörigen Derivate sind Chinosol (o-oxychinolinsulfosaures Kali), Chinaseptol s. Diaphtol (0-Oxychinolinsulfosäure), Oxychinaseptol s. Diaphterin, Argentol (Chinaseptolsilber), Loretin (m-Jod-0-oxychinolin-ana-sulfosäure) und V i o f or m (m-Jod-ana-chloro-oxychinolin.)

Schon auf Grund letztgenannter Tatsache, aber mehr noch, weil wir im Chinolin den typischen aromatischen Grundkomplex der Chinaalkaloide ${ }^{1}$, ebenso wie der von Liebig im Hundeharn entdeckten Kynurensäure vor uns haben, besitzt sein Verhalten im Tierkörper auch noch heute hervorragendes Interesse.

Über die physiologisehe Wirkung des Chinolins liegt schon aus dem Jahre 1874 eine allerdings an unreinem Präparate ausgeführte Untersuchung von Mc. Kendrick und J. Dew ar ${ }^{2}$ ) vor. Eine genauere Prüfung verdauken wir A. Biach und G. Loimann ${ }^{3}$, ferner F. Oschatz ${ }^{4}$, J. Rosenthal und seinen Schülern ${ }^{5-8}$ ),

1) AuBer in den Chinaalkaloiden findet sich der Chinolinkern wabrseheinlich noch im Strychnin, Brucin, Curarin und Gelseminin.

2) Mac Kendrick u. J. Dewar, Pbysiolog. Wirkung d. Chinolin- und Pyridinbasen. Referat in Ber. d. Deutsch. chem. Ges. 7, 1458 (1874).

3) A. Biach u. G. Loimann, Versuche über die physiologische Wirkung des Chinolins. Virchows Archiv 86, 456 (1881).

4) F. Oschatz, Experiment. Untersuchnngen ü. d. physiologische Wirkung des Chinolins. Diss. Göttingen 1882.

5) J. Rosenthal, Über die Wirkung des Chinolins. Beitr. zur path. Anatomie, von Zenker gewidmet. Leipzig 1887, S. 207 und Deutsch. Arch. f. klin. Med. 42, 206 (1888).

6) O.R i eger, Versuche ü. d. physiol. Wirk. d.Chinolins. Diss. Erlangen, 1888.

7) M. Jakobsohn, Beitrag z. Kenntnis d. antibakteritischen Eigenschaften d. Chinolins. Diss. Erlangen 1890.

8) G. Wittigschlager, Beitrag z. Kenntnis d. physiol. Wirkungen d. Chinolins. Diss. Erlangen 1890. 
und endlich R. Stockman'). Donaths diesbeztugliche Arbeiten wurden bereits erwähnt.

Donath war zugleich der erste, welcher die Ausscheidungsverhältnisse des Chinolins aus dem Tierkörper zu erforschen suchte. Er konnte feststellen, daß nach Chinolineingabe sich kein freies Chinolin im menschlichen Harn findet und nahm darnm Oxydation desselben im Organismus zu Pyridinkarbonsäure an, indes hat er eine solche Säure ans dem Harn nicht isoliert.

Wenig später, wie Donath, hat sich L. Brie ger ${ }^{2}$ ) mit den Stoffwechselprodukten des Chinolins beschäftigt. Derselbe gibt an, daß sich in dem Harn mit Chinolin behandelter Patienten eine Substanz findet, die mit Bromwasser einen flockigen Niederschlag bildet, welchen er aber in analysierbarem Zustande nicht erhalten konnte. Ferner, daß sich ans dem Harn, nach dem Kochen mit Salzsäure, durch Äther ein prachtvoller roter Farbstoff ausschütteln läßt, der durch Benzol und Eisessig von den übrigen Harnfarbstoffen getrennt werden kann, aber keine Neigung zur Kristallisation zeigt.

In einer auf J. Rosenthals Anregung von G. Wittigschlager(l. c.) ausgeftubrten Untersuchung wurde festgestellt, daßsich aus dem alkaliseh gemachten Harn durch Wasserdampfdestillation und Extraktion des Destillates mit Äther eine honigartig riechende, kristallisierbare, basische Substanz isolieren läBt. Endlich machte E. Rost ${ }^{3}$ ) wahrscheinlich, daß sich das Chinolin im Harn in Form einer mit Sehwefelsäure gepaarten Verbindung findet.

Im Verlauf meiner eigenen Versuche zur Isolierung der Chinolinprodukte aus dem Harn meiner Versuchstiere (Kaninchen) beobaebtete ich eine bisher unbekannte schöne Farbenreaktion des Chinolinharns, welche dadurch an Interesse gewinnt, daß sie an die Jaffẻsche Reaktion der Kynurensäure und zugleich an die Thalleiochinreaktion des Chinins erinnert. Ich habe die Reaktion in den Berichten der Dentschen ehemischen Gesellschaft bereits kurz beschrieben. ${ }^{4}$ )

1) R. Stockman, The Physiological action of quinoline, isoquinoline and some of their derivatives. J. of Physiol. 15, 245 (1893).

2) L. Bri eger, Über d. antipyret. Wirkung d. Chinolin. tartar. Zeitschr. f. klin. Med. 4, 296 (1882).

3) E. Rost, Über d. Schicksal d. o-Oxychinolins und über die Ausscheidung d. gepaarten Schwefelsäuren im Harn d. Hundes; nebst e. Anhang ü. d. Zusammensetzung d. Chinosols. Arb. a. d. Kais. Gesundheitsamte 15, 288 (1899).

4) H. F ü h n e r, Zur Thallciochinreaktion d. Chinins u. d. Kynurensäureeaktion v. Jaffé. Ber. d. Deutsch. chem. Ges. 38, 2713 (1905). 
Versetzt man den frischgelassenen Harn von Kaninchen, denen man mit der Schlundsonde oder subkutan ein Chinolinsalz beigebracht hat, mit Ammoniak, so findet keine Farbenveränderung statt. Kocht man ihn aber zuerst mit konzentrierter Salzsäure (1 Teil Salzsäure auf 2-3 Teile Harn) bis Dunkelfärbung stattfindet und versetzt nunmehr, nachdem abgekühlt, mit Ammoniak im Überschuß, so färbt sich die Flüssigkeit beim Schütteln von der freien Oberfläche ans erst gelbgrün, später blaugrün. Allmäblich bildet sich dann ein dunkler Niederschlag und nach eintägigem Stehen ist die grüne Färbung der Flüssigkeit meist verschwunden. Chloroform nimmt das grüne Produkt zum Teil auf.

Weit schöner bekommt man die Reaktion, wenn man das sie bedingende Chinolinprodukt erst in reinerer Form darstellt, was am besten in folgender Weise geschieht:

$200 \mathrm{~cm}$ Harn werden mit $50 \mathrm{ccm}$ konzentrierter Salzsäure auf dem Wasserbade auf etwa die Hälfte eingedampft. Unter sorgfältiger Vermeidung alkalischer Reaktion versetzt man mit Natronlauge, bis die Reaktion der Flüssigkeit gegen Lackmuspapier nur noch sehr schwach sauer oder neutral ist, filtriert, wäscht das Filter mit etwas Alkohol nach und schüttelt die erkaltete Flüssigkeit mit Äther aus. Den $\ddot{A}$ ther wäscht man einige Male mit geringen Mengen destillierten Wassers und schüttelt ihn dann mit stark verdünnter Salzsäure. Die Salzsäure nimmt hierbei intensiv gelbe Färbung an, während der überstehende Äther karminrot gefärbt erscheint. Verdünnt man einige Tropfen der gelb gefärbten Salzsäure mit Wasser und versetzt nunmehr mit Ammoniak, so tritt Grünfärbung auf, welche unter der Einwirkung des Luftsauerstoffs rasch in reines Blau übergeht. Bei längerem Stehen verdünnter Lösungen, rascher in konzentrierten, bilden sich dunkelblave amorphe Flocken und setzen sich zu Boden. Diese lösen sich, abfiltriert, in Alkohol mit blauer, in Mineralsäuren mit braunroter oder karminroter Farbe.

Dieselbe Farbenreaktion zeigt der Harn von Hunden nach Chinolingaben, und, wie ich mich durch einen Selbstversuch überzeugte, auch der mensehliche Harn.

Ich nahm in einer Oblate $1 \mathrm{~g}$ reines (schneeweißes) weinsaures Chinolin. Der nach einer Stunde gelassene Harn gab die Reaktion noch nicht, hingegen Harn, welcher nach zwei Stunden gelassen wurde. Zehn Stunden nach dem Einnehmen des Chinolins entleerter Harn zeigte die Reaktion noch sehr deutlich, hingegen nicht mehr der Harn nach zwanzig Stunden.

Das die Grünfärbung bedingende Produkt geht aus neutraler, 
nicht aus saurer Lösung in $\ddot{A}$ ther. Es ist basischer Natur und in der salzsauren Lösung als Salz enthalten. Doch finden sich in der sauren Lösung noch andere Produkte, welche die Reindarstellnng des hier interessierenden Körpers sehr erschwerten. Erschwert wurde dieselbe auch dadurch, daß das Produkt, in Berührung schon mit schwachen Alkalien, sich sofort zersetzt. Nur durch Natriumazetat gelingt es, die Base unzersetzt und in ziemlich reiner Form als braune Flocken, die später kristallinisch werden, auszufällen. Die Ausscheidung findet bei noch saurer Reaktion statt; alkalische Reaktion ist auch hier $\mathrm{zu}$ vermeiden.

Zur Analyse ausreichende Mengen des Produktes konnte ich nicht darstellen und mußte darum auf indirektem Wege, durch Vergleichung mit anderen, durch Alkalien Grünfärbung gebenden Chinolinderivaten, zu einer Identifizierung des Körpers zu gelangen suchen.

Zunächst sei hier die Beobachtung von H. Decker und C. Kop ${ }^{1}$ ) erwähnt, daß durch Oxydation von Additionsprodukten des Chinolins am Stickstoff Indigo erhalten werden kann. Der Harnfarbstoff besitzt jedoch nicht die Eigenschaften, namentlich nicht die Beständigkeit des Indigos.

Hingegen erinnert er an die blau- bezw. grïngefärbten, von Williams entdeckten schönen aber unbeständigen Cyanine, welche gegenwärtig als photographische Sensibilisatoren Verwendung finden.

Cyanine bilden sich bei der Einwirkung von Alkalien auf ein Gemisch von Alkylaten des Chinolins und seiner Homologen.

Vom Pyridin ist durch W. His'2) Untersuehung bekannt, daß es im Tierkörper am Stickstoff methyliert wird. Das vom Pyridin sich nur durch das Plus eines Benzolkernes unterscheidende Chinolin konnte sich ebenso verhalten und in diesem Falle war bei Verfittterung von unreinem Chinolin, welches die zur Cyaninbildung nötigen homologen Produkte enthält, Entstehung von Cyanin oder vielmehr von den zu seiner. Bildung durch Alkali nötigen alkylierten Vorstufen nicht ausgescblossen. Da ich aber absolut reines Chinolin verwandte, war Cyaninbildung unwahrscheinlich.

Cyanine können dargestellt werden aus alkylierten 2- und 4-Methylchinolinen. (Über die Konstitution der Cyanine vergleiche

1) H. Decker a. C. Kopp, Bildung r. Indigo aus Chinolin. Ber. d. Dentsch. chem. Ges. 39, 72 (1906). $253(1887)$.

2) W. H is, Über d. Stoffwechselprodult d. Pyridins. Dieses Archiv 22, 
A. Miethe und G. Book 1).) Es ist nun interessant, daß auch das 2, 4-Dioxychinolin, wie P. Friedländer und A. Weinberg ${ }^{2}$ ) beobachteten, unter der Einwirkung von Ammoniak Blaufärbung zeigt. Diese erscheint aber erst bei längerem Stehen der Lösung, während sie bei dem Harnprodukt sofort auftritt. Grünfärbung durch Alkalien zeigt auch das 2, 3, 4-Trioxyehinolin, hingegen nicht das 2, 3-Dioxychinolin.

Ebenso wie die bisher genannten Produkte im Pyridinkern substitutiert sind, ist es die Kynurensäure (4-0xy-3-chinolinkarbonsäure), welche bekanntlich nach der Behandlung mit Kaliumehlorat und Salzsäure mit Ammoniak Grïnfärbung gibt. ( $J$ af fès Reaktion). Die Karboxylgruppe der Kynurensäure ist zum Zustandekommen der Reaktion nicht erforderlich: Das 4-Oxychinolin (Kynurin) gibt dieselbe in gleicher Weise.

Bei dieser Chlorierung der Kynurensäure findet zugleich Oxydation statt. Es bildet sich, wie Jaffé feststellte, ein Tetrachlordioxychinolin ${ }^{3}$ ), also wiederum ein zweifach oxydiertes Prodakt, das sich durch Ammoniak grün färbt. Während aber mein Harnprodukt Grünfärburng mit Ammoniak beim Zusatz desselben zu seinen $L_{0}$ sungen gibt, konnte ich eine solche bei der Kynurensäure und dem Kynurin nur beim Übergießen des trook en en Chlorproduktes mit Ammoniak erhalten. Auch hier zeigt sich also keine Identität mit dem Verhalten des Harnproduktes.

Doch nicht nur im Pyridinkern oxydierte Chinolinderivate geben mit Ammoniak Grïnfärbung, sondern auch solche, die im Benzolkern oxydiert sind. Zu diesen gehört das Chinin mit seiner charakteristischen Thalleiochinreaktion. Wie Skraup ${ }^{4}$ nachgewiesen hat, ist diese Identitätsreaktion des Chinins zurückzuführen auf den im Chininmolekül enthaltenen p-Chinanisolkomplex. Doch auch das Cuprein zeigt die Thalleiochinreaktion. Da das Cuprein an Stelle der Methoxylgruppe des Chinins die Hydroxylgruppe besitzt, so war es von vornherein wahrscheinlich, daß auch das p-Oxychinolin ge-

1) A. Miethe u. G. Book, Über d. Konstitution d. Cyaninfarbstoffe. Ber. d. Deutsch. chem. Ges. 37, 2008, 2821 (1904) u. 38, 3804 (1905). Vergl. hierzu: W. Koenig, Z. Frage d. Konstitution d. Cyaninfarbstoffe. Journ. f. prakt. Chem. (2) 73, 100 (1906).

2) P. Friedländer u. A. Weinberg, Über einige im Pyridinkern substituierte Chinolinderivate. Ibid. 15, 2679 (1882).

3) M. J a f fé, Eine empfindliche Reaktion anf Kynurensäure. Zeitschr. f. physiolog. Chemie 7, 399 (1882-1883).

4) Zd. H. S kraup, Über d. Parachinanisol. Wiener Monatshefte f. Chemie $6,760(1886)$. 
nannte Reaktion geben werde, eine Vermutung, welehe ich in der Tat bestätigt fand. 1)

Die Thalleiochinreaktion wird bekanntlieh in der Weise angestellt, daß man die Lösung des Chininsalzes mit Chlorwasser und nachher mit Ammoniak versetzt. Beim p-Oxychinolin bildet sich dureh die Chloreinwirkung ein 5,5-Dichlor-6-ketochinolin(II), worüber

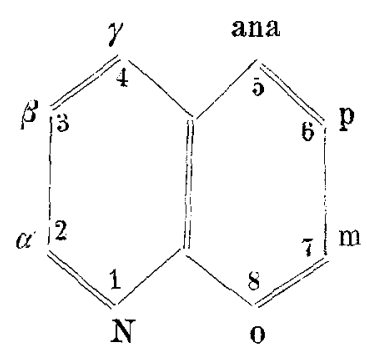

Chinolin

I

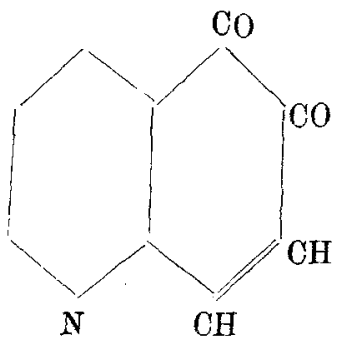

5,6-Chinolinchinon

III

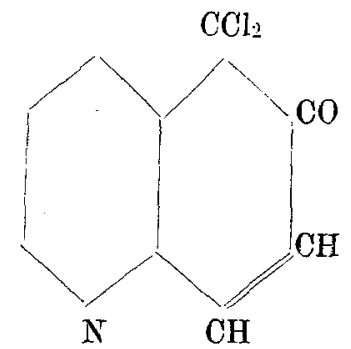

5,5-Dichlor-6-Ketochinolin

II

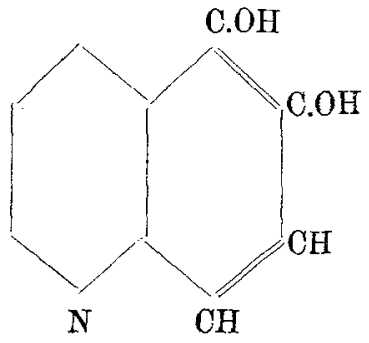

5,6-Dioxychinolin

IV

ich ausfübrlicher an anderer Stelle berichten werde. Dieses Produkt gibt mit Ammoniak auch in seinen Lösungen sehr schöne Grünfärbung, und der beim Stehen ausfallende dunkelblaue Farbstoff zeigt große Ähnlichkeit mit dem Harnfarbstoff, ist auch wie dieser halogenfrei. Aber abgesehen davon, daß die Bildung eines solchen Chlorproduktes im Tierkörper unwahrseheinlich ist, deekt sich das Verhalten des Dieblorketons durehaus nieht mit demjenigen des braunen, aus dem Harn erhaltenen Produktes.

Dem Dichlorketochinolin in seiner Konstitution analog gebaut

1) H. Fühner, 1 c.

Archiv f. experiment. Pathol. u. Pharmakol, Ba. LV. 
ist das von Mathëus 1) dargestellte 5, 6-Chinolinchinon (III) und dieser Körper verhält sich in seinen gelbgefärbten, salzsauren Lösungen der Einwirkung von Ammoniak gegenüber genau wie das Harnprodukt. Die Lösung wird durch Ammoniak grün und färbt sich, wie der Autor angibt, „rasch von oben her tiefblau“.

Die Angaben von Mathëus tiber die Eigenschaften des Chinolinchinons sind aber so spärlich, daß ich nach denselben die Identität von synthetischem Produkt und Harnprodukt wohl vermuten, doch nicht sicher feststellen konnte. Es war darum eine direkte Vergleichung beider Produkte erforderlich, welche leicht die völlige Übereinstimmung des Harnproduktes mit dem 5,6-Chinolinchinon ergab.

Der Beschreibung von Mathëus kann ich auf Grund meiner Beobachtungen folgendes binzufügen:

5, 6-( $\beta$-Chinolinehinon. Man erhält das Chlorhydrat des 5,6-Chinolinchinons nach den Angaben von Mathëus durch Oxydation des 5-Amino-6-oxychinolins mit Eisenchlorid. (Vgl. zur Darstellung und den Eigenschaften dieses Produktes auch Th. Zincke und K. Wiederhold ${ }^{2}$ ).) Das freie, bisher unbekannte Chinon läßt sich erhalten durch Fällung der wässerigen Lösung des Chlorhydrats vermittelst Natriumazetat. Die Ausscheidung des freien Chinons erfolgt in Form brauner Flocken, bei längerem Stehen in kristallinischer Form und zwar schon bei saurer Reaktion der Lösung (gegen Lackmus).

Das Chinolinchinon kristallisiert in zu Gruppen vereinigten glänzenden Prismen. Größere Kristalle erscheinen dunkelbraun gefärbt, mikroskopisch kleine nahezu farblos. Das kristallinische Produkt ist in Wasser und organischen Lösungsmitteln sehr schwer löslich. Die verdünnten Lösungen sind farblos und färben sich bei längerem Stehen an der Luft rosa bis violett. Leichter löst sich das Chinon in Eisessig und Mineralsäuren mit typiseher Chinonfarbe.

Das Produkt ist geruchlos und mit Wasserdämpfen nicht flïchtig, schließt sich also in seinem Verhalten dem $\beta$-Naphtochinon an. Es schmilzt auf dem Platinblech. Im Kapillarrohr ist auch bei raschem Erhitzen bis zur Temperatur von $350^{\circ}$ kein Schmelzen zu beobachten. Bei trockenem Erhitzen der Substanz beobachtet man iber der Erhitzungsstelle die Bildung eines grünen Sublimats.

1) J. Mathëus, Über ein neues Chinolinchinon. Ber. d. Deutsch. chem. Ges. 21, 1887 (1888).

2) Th. Zincke u. K. Wiederhold, Über Dichlor- $\beta$-chinolinchinon und dessen Umwandlungsprodukte. Liebigs Annalen d. Chemie 290, 359 u. 364 (1896). 
Das $\beta$-Chinon ist bedeutend stärker basisch, als das von $0 . \mathrm{Fi}$.

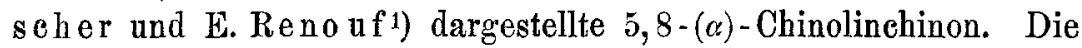
Lösungen seiner Salze werden durch Wasser nicht dissoziiert und die Base läßt sich nur in neutraler oder kaum saurer Lösung mit Äther ausschütteln. Man versetzt, um das Chinon auszuschütteln, die reingelbe Lösung des Chlorhydrats mit stark verdünnter Natronlauge vorsichtig tropfenweise, bis die Farbe der Lösung rotbraun geworden ist und äthert dann aus. Nach dem Verdampfen des farblosen, mit Wasser gewaschenen Äthers im Vakuum hinterbleibt ein gelber mikro-kristallinisoher Rückstand, welcher aus einer Mischung von gelben und fast farblosen Kristallen besteht. Letztere stellen die freie Base dar, erstere besitzen Salzform und sind in Wasser löslich.

Versetzt man die absolut alkoholisehe Lösung von Base oder Salz mit einer Spur Ätzkali, so beobachtet man eine sehr raseh vorübergehende Grün-, dann Violettfärbung, welche bald durch Rot in Gelb tibergeht. Der Farbenwechsel entspricht, abgesehen von der Grüfärbung der Bambergersehen Reaktion der cyklisehen $\alpha$-Diketone.

Mathëus beobachtete Grünfärbung der Chinonsalzlösungen nur durch Ammoniak. Entgegen seinen Angaben läßt sich durch verdünnte Natronlange, Natriumkarbonat, Barytwasser usw. dieselbe Grünfürbung erhalten, welche aber sehr rasch vorübergeht und nur beim Ammoniak beständig ist. Ich möchte die erstauftretende Grünfärbung für eine Chinhydronbildung ansprechen, auf welche dann beim Ammoniak Bildung eines Chinonimidfarbstoffes folgt. Das durch Ammoniak ausfallende amorphe blaue Produkt löst sich in Mineralsäuren mit roter Farbe. Versetzt man die saure Lösung jetzt mit einem beliebigen Alkali, so erhält man wieder den blauen Farbstoff. Über diesen werde ich anderorts berichten.

Es sei an dieser Stelle erwähnt, daß ich auch an einer alkoholisch-wässerigen Lösung von $\beta$-Naphtochinon (Präparat von Th. Schuchardt, Görlitz) beim Überschichten mit Ammoniak schöne Grünfärbung beobachtete. Vielleicht zeigen noch andere aromatische $\alpha$-Diketone diese Reaktion.

Sehr verdünntes Eisenchlorid erzeugt in der neutralen, wässerigen Lösung des Chinolinchinonçhlorhydrats Grünfärbung. Dieselbe vergeht beim Erwärmen und erscheint beim Erkalten wieder. Weiterer

1) O. Fischer u. E. Renouf, Über einige Abkömmlinge d. o-Oxychinolins. Ber. d. Deutsch. chem. Ges. 17, 1642 (1884). 
Zusatz von Eisenchlorid bringt die grüne Farbe zum Versehwinden. Versetzt man jetzt mit Ammoniak, so tritt die typische Blaufärbung auf.

Die Beobachtung spricht wohl für gleichzeitiges Vorhandensein von etwas Dioxychinolin in der Chinonlösung.

Ein sehr charakteristisehes Derivat des $\beta$-Chinolinchinons erhält man durch Einwirkung von Anilin auf dasselbe. Eine Lösung des Chinons in Essigsäure kondensiert sich leicht sehon in der Kälte mit überschüssigem Anilin zu einem chlorophyllgrün gefärbten A nilid, welches in Flocken ausfällt, die aus einem Haufenwerk feiner Nadeln bestehen. Die Flüssigkeit färbt sich dabei rosarot. Auf der Oberfläche derselben beobachtet man manchmal eine dünne kupferrote Kristallhaut. Beim Abfiltrieren der grünen Flocken haften ihnen die nur in geringer Menge vorhandenen kupferroten Kristalle an und̉ täusehen roten Oberflächenglanz des grtinen Produktes vor, da sie sich auch durch Auswaschen nicht entfernex lassen. Das grüne Anilid ist in allen gebräuchlichen Lösungsmitteln unlöslich. Konzentrierte Schwefelsäure löst es mit karminroter Farbe, welehe rasch in Violett übergeht. Beim Verdünnen mit Wasser tritt Entfärbung ein. Hinsichtlich seines Schmelzpunktes verhält sich das Anilid wie das Chinon selbst.

Wie erwähnt, färbt sich die saure Flüssigkeit bei der Kondensation rosarot. Versetzt man mit Alkali und treibt das Anilin mit Wasserdämpfen $a b$, so hinterbleibt ein brauner, in verdtunnten Säuren mit fuchsinroter Farbe löslicher Rückstand, welcher aus der sauren Lösung durch Alkalien in Form rotbrauner Flocken ausgefällt wird. Das trockene Produkt löst sich in konzentrierter Schwefelsäure mit grüner Farbe auf, welche beim Verdünnen mit Wasser in Rot iibergeht. Es scheint also bei der Kondensation des Chinons mit Anilin als Nebenprodukt ein Azin gebildet zu werden.

$\mathrm{Zu}$ einem Azin von den eben beschriebenen Eigenschaften gelangt man auch durch Kondensation des Chinons in essigsaurer oder ätherisch-alkoholischer Lösung mit einer alkoholischen Lösung: von $m$-p-Toluylendiamin.

Reduktion der ätherisch-alkoholischen Lösung des Chinons durch schweflige Säure zu dem entsprechenden Hydrochinon (Dioxychinolin) gelang mir nicht.

Recht auffallend ist bei dem von mir dureh Fällung mit Natriumazetat erhaltenen Chinolinehinon seine geringe Löslichkeit in organischen Lösungsmitteln und sein hoher Schmelz- bezw. Zersetzungspunkt, ein Verhalten, das sehr an das der bekannten Dioxy- 
ehinoline erinnert. Vielleicht besitzt das braune, kristallinische Produkt die Konstitution eines Dichinolyls. Für eine Veränderung in der Konstitution des arsprünglichen Chinons würde auch folgende Beobachtung sprechen:

Löst man das braune Produkt in heißer verdünnter Salzsäure kalt ist es sehr schwer löslich - und versetzt nach dem Abkühlen mit überschüssigem Ammoniak, so scheiden sich nach längerem Stehen der grïnen Lösung chlorophyllgrüne amorphe Flocken ab. Abgesehen davon, daß dieser Niederschlag nicht die Blaufärbung: des aus dem ursprünglichen Chinolinchinonchlorhydrat erhaltenen Produktes besitzt, löst er sich in Säuren nicht mit roter, sondern mit gelbbrauner Farbe. Doch möchte ich auf diese Beobachtung deshalb kein zu großes Gewicht legen, weil alle diese blauen Ammoniakniederschläge, wenn einmal gebildet, zwar relativ beständig sind, zu ihrer Bildung aber von verschiedenen günstigen Umständen abhängen.

In dem frischgelassenen Harn der Versuchstiere ist das Chinolinchinon als solches noch nicht enthalten. Man muß annehmen, daß es in reduzierter Form, als 5, 6-Diox yehinolin (IV), mit Schwefelsäure oder Glykuronsäure gepaart, durch die Niere ausgeschieden wird. Durch Koch en mit Salzsäure wird die gepaarte Verbindung zerlegt und das 5,6-Dioxychinolin, als Hydrochinon wenig beständig, oxydiert sich zum entsprechenden Chinon.

Ich konnte im Harn der Versuchstiere in der Tat eine Vermehrung der geparten Schwefelsäure nach Chinolinfütterung nachweisen, und demnach die Vermutung von Rost ${ }^{1}$ ) bestätigen, konnte aber noch nicht feststellen, ob gerade das hier beobachtete Produkt mit Schwefelsäure gepaart wird.

Das Chinolin wird somit im Tierkörper ebenso, wie ich dies für das Akridin ${ }^{2}$ ) feststellen konnte, oxydiert und zwar wie letzteres in p-Stellung zum Stickstoff. Die einmal in dieser Stellung eingetretene Oxydation erleichtert dann weitere Oxydation in anaStellung ${ }^{3}$ ). Chinolin, wie Akridin folgen also der zuerst von

1) E. Rost l. c.

2) H. Fühner, Über das Verhalten d. Akridins i. Organismus d. Kaninchens. Dieses Archiv 51, 391 (1904).

3) Die negative $\mathrm{OH}$-Gruppe in der 6-Stellung wirkt orientierend bei weiterer Substitution und zwar in der Weise, dafs eine eintretende negative Gruppe die benachbarte 5-Stellung besetzt. So bildet sich bei der Chlorierung des p-Oxychinolins zuerst ein 5-Chlor-6-Oxychinolin (Zincke), bei der Behandlung mit salpetriger Säure ein 5-Nitroso-6-Oxychinolin (Mathëus); genau ebenso hier im Tierkörper ein 5,6-Dioxychinolin. 
Schmiedeberg ${ }^{1}$ ) am Anilin beobachteten Gesetzmäßigkeit, daß Oxydation im Tierkörper in p-Stellng zum Stickstoff stattfindet.

Der p-Stellung im Benzolkern des Chinolins entspricht die $\gamma$-Stellung im Pyridinkern. In $\gamma$-Stellung oxydiert ist die Kynurensäure. Wie Ell ing e ${ }^{2}$ ) nachweisen konnte, entsteht Kynurensäure im Tierkörper bei der Fütterung mit Tryptophan, einem Indolderivate, auf oxydativem Wege. Hierbei findet Oxydation in $\gamma$-Stellung zum Stickstoff statt. p- und $\gamma$-Stellung besitzen demnach als Angriffspunte physiologischer Oxydation ähnliche Dignität, was natürlich in innigem Zusammenhang mit den Bindungsverhältnịssen der Kohlenstoffatome im Chinolinmolekül steht.

Zum Schlusse möchte ich auf die biologiseh interessante Tatsache binweisen, daß p- und $\gamma$-Stellung ebenso wie für den Tierkörper anch für die Pflanze als Angriffspunkte von Bedeutung sind, denn in den Chinaalkaloiden sind auch gerade diese beiden Stellungen im Chinolinkern substituiert.

Das 5,6-Chinolinehinon bezw. Hydrochinon ist wohl das auffallendste, aber nicht das einzige Produkt, in welches das Chinolin im Tierkörper umgewandelt wird. Es finden sich neben diesem im Harn der Versuchstiere noch mindestens drei weitere Produkte, auf welche ich später zurückzukommen hoffe.

1) O. S c h m i e d e b e r g, Über d. Verhältnis d. Ammoniaks u. d. primăren Monaminbasen z. Harnstoffbildung i. Tierkörper. Dieses Archiv 8, 1 (1878).

2) A. Ellinger, Die Entstehung d. Kynurensäure. Ber, d. Dentsch. chem. Ges. 37, 1801 (1904) u. Zeitschr. f. physiol. Chem. 43, 325 (1904-1905). 